\title{
3D Measurement of Children - Shape GB - The UK National Childrenswear Survey
}

\author{
Richard Barnes, Select Research Ltd, UK
}

\section{Introduction}

Shape GB is the first phase of a major national research project to measure children, seven years since the last National Sizing Survey measured men and women as part of Size UK.

The first phase of Shape GB was funded by major clothing retailers including Next, Monsoon, Shop Direct and George at ASDA and supported by The University of Hertfordshire, Aston University, Loughborough University, Manchester Metropolitan University and The University of Hull. The research was co-ordinated and managed by Select as the established specialists in this large scale sizing surveys in the UK. A key element of the research approach was the use of 3D body scanning technology to successfully capture the data in place of more traditional methods, measuring over 2,500 boys and girls aged $4-17$. It is the first large scale survey for the retail clothing industry in the UK on children since 1978 and the first using 3D scanners.

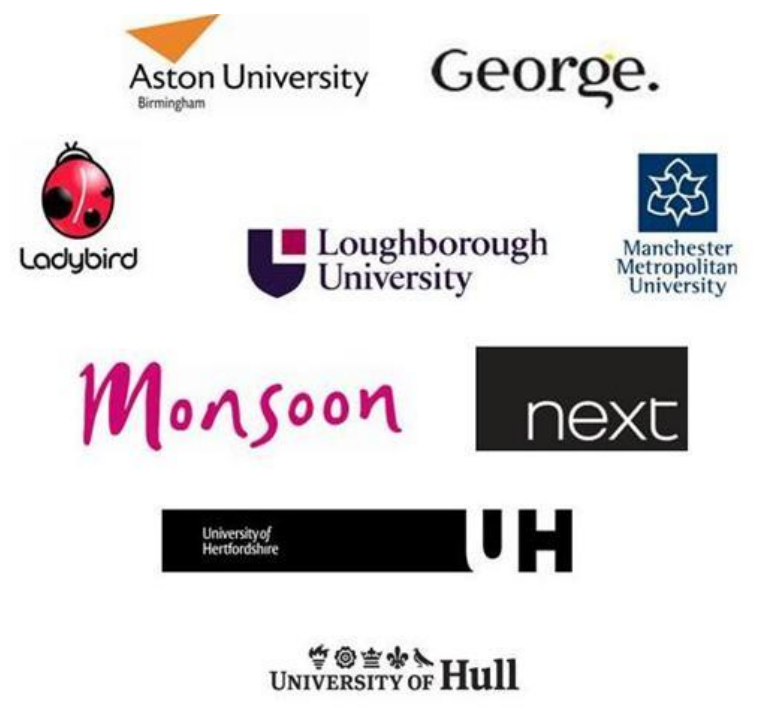

Fig. 1. Collaborators on Phase1 of Shape GB - The National Childrenswear Survey

The second phase of the research, which commences in Autumn 2011, will aim to repeat the first phase but with a focus on the measurement of babies and toddlers and is funded by additional retailers committed to the Shape GB project. The third phase in 2012 will concentrate on ensuring that national representation on ethnicity is achieved in line with current census data.

For Phase one, over an 18 month period from March 2009 to August 2010, more than 2,500 children aged 4-17 were scanned at twelve locations across England, Scotland and Wales, to obtain a representative sample of UK children. $[\mathrm{TC}]^{2} \mathrm{NX12} 3 \mathrm{D}$ body scanners were used to collect 3D scans of the children together with a small number of supplementary manual measurements.

The data collected provide a robust 3D data set, the first set ever available on children. At present the data contain nearly 200 individual measurements encompassing lengths, girths and circumferences that provide not only the familiar measures of body size, but also provide a much greater understanding of body shape and the changes in children over the years. 


\section{History and Development}

The use of scanning technology for this form of data collection has a number of significant benefits and as is well documented, having been used predominantly for the measurement of adults over the past twenty years. As the primary data collected from a 3D image gives a 3D surface representation of the body, measurements can be extracted from this body model at any time and the same applies to children. Thus, the standard measurements used for adult data analysis may be supplemented at any time for different purposes with additional measures to meet user needs. These additional measures can be extracted from the body models in an automated fashion with the fundamental benefit of not having to re-measure participants manually.

The capture of a 3D body model also allows an understanding of body form or shape much greater than that afforded by most existing data sources, which predominantly contain a series of discreet 2D measurements and so are particularly relevant to children, as body shape changes as they get older. 2D measures can still be obtained from the scans where desired, however the scans can also be used to create 3D surface mannequins, either individually, or as some form of boundary mannequin that can be created to represent useful multivariate representations of body size and shape and support a wide variety of applications.

Select were involved in the management and set up of the 1999 childrenswear survey for M\&S which involved measurement of over 4,000 children aged 0-16. However, as the first major survey to involve scanning children, the process of conducting the Shape GB survey was also a challenging task. Ethical procedures were established and approved by each institution and recruitment of children with parental consent was undertaken via the project website ${ }^{1}$ which provided information about the project and allowed parents to book their children in to be scanned at the nearest scanning location. A lot of care was taken to provide clear information to both parents and children about the process, and to what the children and parents could expect.

The children who took part were amongst the first children in the world to be measured on this scale using a 3D body scanner, so the initial interest was high.

Issues of privacy and confidentiality were clearly a critical part of the data collection process. Children were scanned in their underwear so parental supervision was essential, together with careful management at the scanning location to avoid any lack of privacy. As the body scanners work on contrast, the child's underwear had to be a similar colour to their skin colour, so uniform underwear was provided to the project by Next. George at ASDA supplied all boys and girls dressing gowns.

On the Size UK project amongst adults, Select were responsible for the management of the recruitment and sampling for the national survey, so to maintain continuity for the subsequent national survey on children, Tc2 scanners were again used which being similar to a photo booth and with a changing room attached, were not inhibiting to children. This arrangement allows the child to get undressed, be scanned, and get dressed again all without having to leave the scanner. The scanning process itself takes approximately 6 seconds and uses white light that appears as a series of white striped projections to the child being scanned. Children were shown the scanner in operation before being scanned, which gave them an opportunity to opt out of the process if they wished.

All the data captured was stored anonymously and in addition, even though the body model is a detailed 3D representation of the child's body, the scan is not recognisable. Figure 2 gives an example of what a child's scan may look like after the measurement information has been processed. The final version may or may not include a 'generic' head attached which will make the image seem more 'real', but avoids any chance of the individual being recognised. 




Fig. 2. An example of an 8-year old boy and extracted measurements

Figure 2 also illustrates some of the shortcomings of using this form of 3D body scanner. The scanner does not fully capture extremities, so manual measures need to be taken to supplement the scanned data.

After a child was scanned, a trained male or female researcher with Criminal Records Bureau (CRB) clearance collected stature, weight, head circumference and a series of hand measurements using manual methods.

Even with the efforts of the research team, understandably there was some anxiety amongst parents, especially in view of the fact that children were to be scanned in their underwear. Below are a number of issues that were pre-empted and how some of their questions were addressed:

\section{Can I go into the scanner with my child?}

You will be able to go into the scanner initially to familiarise your child with the surroundings, but while the scan is taking place a curtain will be drawn and your child will be alone in the booth. You will be able to stand outside the curtain to re-assure your child.

\section{Are there any disadvantages or risks of taking part?}

There are no side effects to using the 3D body scanner. Clothing manufacturers have scanned thousands of adults in the past. However during the scanning there are several brief bursts of flashing lights which can affect people who experience seizures. If your child/ren have had seizures or are on treatment for seizures you must let us know.

\section{What happens when the survey ends?}

Once the survey is complete, we will collate all the scans we have collected and the information will be used when designing, specifying and manufacturing clothing. Given the national representation of the sample, in the long-term the anonymous data will also be used for other purposes such as seat design, healthcare statistics and to analyse height variation between children of the same age. 


\section{Will my child's taking part in the survey be kept confidential?}

Yes. We will follow the Market Research Society Code of Conduct, the Data Protection Act and legal practice. All information about your child will be handled in confidence.

The process of being measured for Shape GB resulted in an almost universally positive experience for those involved and there were no complaints from the 2,500 children or parents about the scanning process. Children genuinely seemed to enjoy the experience, and all participants were engaged with the notion of being able to improve the understanding of body size. From discussion with parents during the data collection, it was clear that parents were well aware of the implications of clothing retailers working with out-of-date data and rarely did parents report that they could actually buy clothes in the appropriate size (age) range for their child.

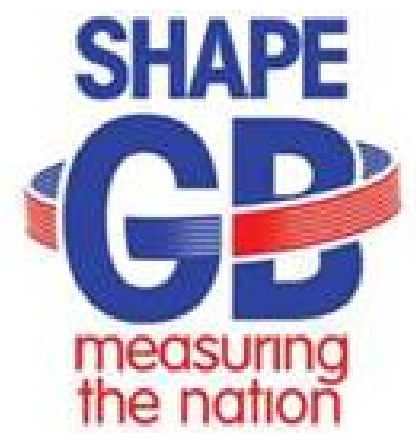

Fig. 3. The logo for Shape GB designed to reflect the Great Britain geographical region where respondents were recruited and measured (England, Scotland \& Wales). The colours reflect the British flag and the 'GB' branding is already closely associated with the 2012 Olympics

The data gathered provides a more sophisticated means of quantifying and analysing the size and the shape of children of different ages. Whilst this was primarily driven by the needs of the retail clothing sector, it has clear implications and applications in other industries where out-dated data for children is currently being used to assess and deliver applications.

UK retailers are now working with and starting to harness the applications of using the data, but the benefit of the data for practitioners, following initial analysis, is now starting to become apparent. As an example of new insights, the results from Shape GB show that the average right thigh girth in boys and girls is always bigger than the left; a general assumption hitherto not proven.

Across all yearly age groups for both boys and girls, the average right thigh for boys is $0.45 \mathrm{~cm}$ bigger in circumference than the left and the average right thigh for girls is also $0.42 \mathrm{~cm}$ bigger than the left.

Analysing data from the 2,500 3D scans (Fig.4), this is the first time that differences between the limbs for children have been scientifically and independently measured and the new data supports the anecdotal evidence that approximately $81 \%$ of people are right footed ${ }^{2}$. This provides an indication of the potential for future measurement of muscle development in children, which has implications for fields such as sports science in the future and illustrates how comprehensive 3D data can provide new insights.

The data also showed differences between the right arm and left arm, with the average right bicep measurement again being bigger than the left in both boys and girls. Biceps in girls are $3.1 \%$ bigger than boys in the early years (4-7) but when boy's biceps start to get bigger as their muscles develop, in teenage years (13-17) the trend is reversed and girl's biceps are then 3.3\% smaller than boys, a swing of over $6 \%$. In some ways obvious, given that a majority of children are right handed or footed, but never measured properly before amongst so many subjects and using 3D. 


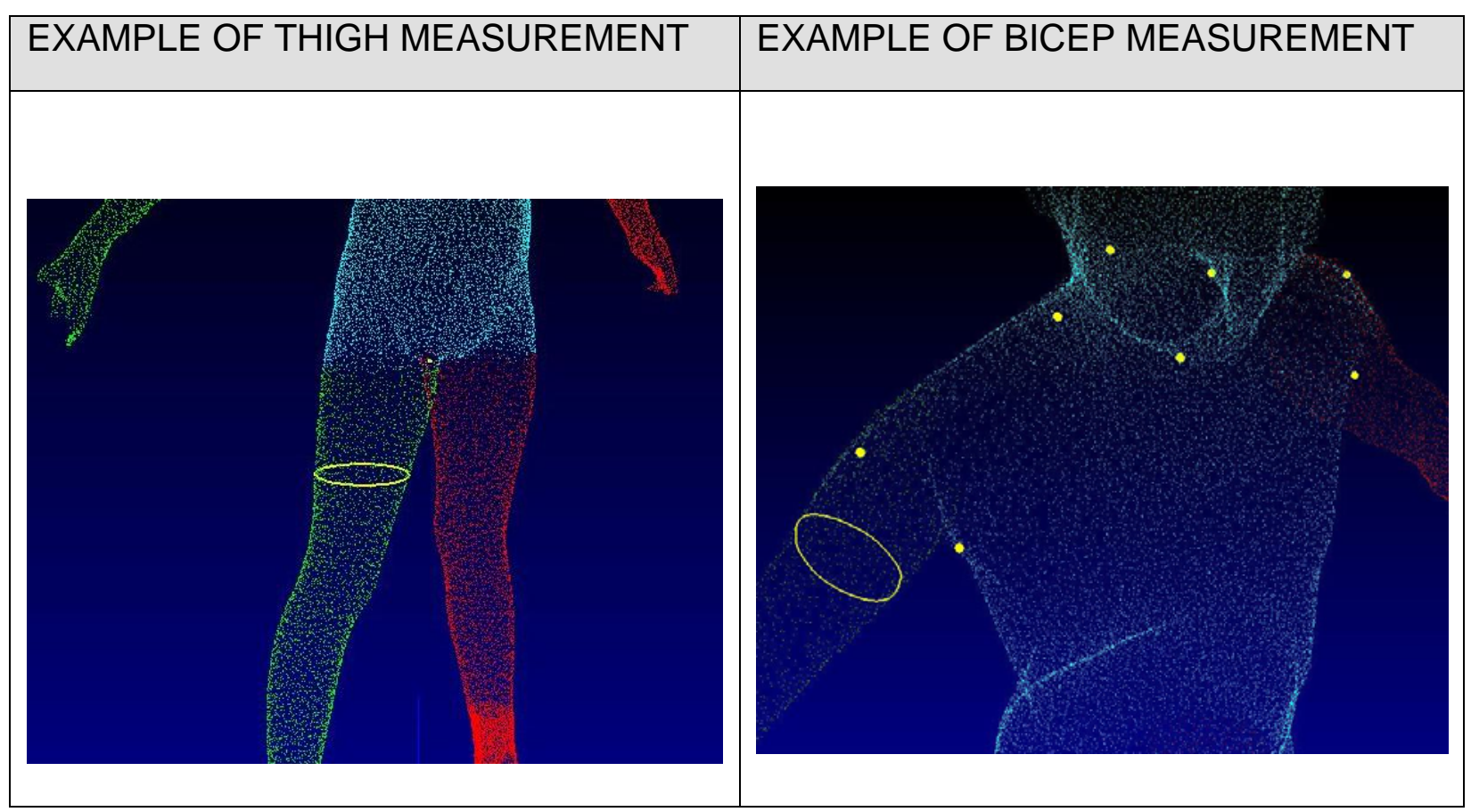

Fig. 4. Examples of the thigh and bicep measurements taken from the scanner

The images above illustrate that 3D data can provide insights into children's body shape, not possible with existing data sources. Product, environment and workplace design relies on accurate and usable data, particularly for areas such as custom fit products and products aimed at improved task or sports performance. This new data provides alternative options of measuring and understanding how children change as they grow older, their changing needs for products but also the effect of different activities and sports on a child's physique.

Key findings for garment design and labelling from Shape GB were presented at a retail clothing industry conference in May 2011, but data is also currently being analysed for future measurement of childhood obesity using the new obesity measurement; the Body Volume Index. Key collaborators in the development of BVI are working together to ensure that the changes in body shape for children of different ages are properly assessed. This is particularly significant for the National Health Service (NHS) and the UK Government whose definitions of childhood obesity are currently formulated using manual data from the 1970's. Shape GB has proven that children have increased in size and shape since the last publicly available data from 1978 and 3D data will provide healthcare professionals with an additional dimension hitherto not available.

In the field of ergonomics, industry standards, such as British Standard EN- $1729^{3}$ for classroom furniture in all UK schools are just one indication of how manufacturers are having to adapt their production and design processes to account for the fact that children have become larger.

The academic and commercial collaborators on Shape GB have been instrumental in successfully completing the first 3D survey on children's body shape and the purpose of the project was to create a new awareness and usage of up to data on children. The importance of being able to apply 3D data on children to retail, healthcare and ergonomic applications is in its infancy but will help to address some of the key issues in those and in other areas.

\section{References}

1. www.shapegb.org

2. C. Porac and S. Coren. Lateral preferences and human behavior. New York: Springer-Verlag, 1981

3. http://shop.bsigroup.com/en/ProductDetail/?pid=000000000030152602 